\title{
LA MUERTE EN LAS PUBLICACIONES CIENTÍFICAS CUBANAS, PERÍODO 2012-2017. RESULTADOS PRELIMINARES
}

DEATH IN CUBAN SCIENTIFIC PUBLICATIONS:

PERIOD 2012-2017. PRELIMINARY RESULTS

Recibido: 12 de febrero de 2018 | Aceptado: 09 de abril de 2018

DOI: 10.22199/507187475.2018.0002.00005

\section{ANTONIO RODRÍGUEZ SÁNCHEZ A, ESTRELLITA FORNÉS BÁEZ B, SANDRO BARÓ LLINS B}

A. hospital universitario general Calixto garcía, La Habana, Cuba;

B. UNIVERSIDAD DE LA HABANA, La Habana, Cuba

\begin{abstract}
RESUMEN
La muerte como evento vital al igual que el nacimiento, es uno de los procesos bio- antro-psico-sociológicos 1 de mayor capacidad de impacto en la historia del ser humano. La conciencia de muerte en tanto cogito ${ }^{2}$ que pretende el autoengaño se ha instalado en disímiles producciones culturales, dígase desde las científicas hasta las artísticas, pasando por las prácticas ordinarias. A grosso modo este es el punto de partida para la pertinencia del estudio de la muerte desde su abordaje en el ámbito de la ciencia, específicamente su presencia en las publicaciones cubanas durante el período 2012-2017. Para ello se optó por el estudio bibliométrico de 7 revistas cubanas que representan distintas disciplinas e instituciones científicas con el objetivo de caracterizar la presencia de la muerte en las publicaciones científicas locales, encontrándose un índice de concentración temática, en relación a la totalidad de publicaciones registradas (1227), de un $4 \%$ (49) lo cual indica la baja productividad de estas revistas durante el período comprendido, alertando sobre el hecho fáctico de que la muerte no constituye un objeto de investigación con capacidad potencial para la apertura de nuevas zonas de sentido ${ }^{3}$ que ofrezcan visiones distintas sobre lo humano.
\end{abstract}

PALABRAS CLAVE: Muerte, revistas, publicaciones, productividad.

\begin{abstract}
Just as birth, death constitutes a bio-anthro-psycho-sociological process which has impacted human history tremendously. The awareness of death as a cogito that claims self-deception has been installed in dissimilar cultural productions, ranging from scientific to artistic, through to ordinary practices. In a broad sense, this is the starting point for the relevance of the study of death from its approach in the field of science, specifically its presence in Cuban publications during the period 2012-2017. 0bjective: To characterize the presence of death in local scientific publications. Method: A bibliometric study of seven Cuban journals from different disciplines and scientific institutions was chosen. Results: An index of thematic concentration of 4\% (49 out of 1227 registered publications) indicates low productivity in scientific journals during 2012-2017. Conclusion: As an object of research, death does not have the potential for the opening of new areas of meaning to provide different perceptions of the human phenomenon.
\end{abstract}

KEY WORDS: Death, journals, publications, productivity.

Correspondencia con los autores:

A. Hospital Univer sitario "General Calixto García", Cuba. E-mail: tonypsico14@gmail.com

B. Institución: Dirección de Información de la Universidad de La Habana, Cuba. E-mail: estrellita@dict.uh.cu; sandro@dict.uh.cu

\footnotetext{
1. Este tipo de neologismo es ampliamente usado por Edgar Morín en distintos momentos (tomos) de El Método.

2. "Hace referencia al pensamiento, que emerge de las operacione s computantes de la máquina cerebral, pero que no puede reducir se a ellas. Retroactúa sobre estas computaciones, las utiliza, las de sarrolla y las transforma formulándose en el lenguaje." (Morín, E,. 2003)

3. Concepto creado por el psicólogo cubano Dr. Fernando González Rey, con el cual "designa aquellos escenarios inteligibles que se producen en la investigación científica y que no agotan la cuestión que significan, sino por el contrario, abren la posibilidad de seguir profundizando en un campo de construcción teórica."
} 
La muerte como evento vital al igual que el nacimiento, es uno de los procesos bioantro-psico-sociológicos de mayor capacidad de impacto en la historia humana. La conciencia de muerte en tanto cogito que pretende el autoengaño se ha instalado en disímiles producciones culturales, dígase desde las científicas hasta las artísticas, pasando por las prácticas ordinarias; remite necesariamente a un proceso de reactualización de las necesidades más instintivas: la de supervivencia y conservación de la especie. Pero también y porque no, a aquellas que como seres culturales nos determinan, embúllese y piense, las de trascendencia, las de autorrealización, etc., registrando en el inconsciente colectivo e individual una estructura tanatofóbica que cada vez más se encuentra solapada de contenidos simbólicos que nos movilizan hacia una existencia, más por la ansiedad ante la muerte que por el propio goce que reporta la vida en sí misma en condiciones de su estructura cíclica. "La concepción de la muerte en una sociedad compromete globalmente a todo su sistema cultural. La gestión del proceso de morir, el tratamiento de los muertos o el desarrollo de los rituales relacionados con la muerte conforman una mirada concreta sobre esta, pero también informan sobre la realidad social en su conjunto y sobre la relación de esa sociedad con el mundo." (Jiménez, A., R., 2009).

Obras capitales como "El Hombre y la Muerte", abordada desde una perspectiva antropológica por Morín, E. (2003), que suscita una gran contradicción gnoseológica cuando plantea que "existe, pues, una conciencia realista de la muerte incluso en la noticia prehistórica y etnológica de la inmortalidad: no la conciencia de la esencia de la $<<$ muerte $>>$, ya que esta nunca ha sido conocida ni lo será puesto que la muerte no tiene <<ser»>". "Historia de la muerte en Occidente. Desde la Edad Media hasta nuestros días" del historiador francés
Philippe A. ${ }^{4}$ (1975), en donde propone toda una caracterización de las actitudes hacia la muerte a partir de la relación con la estructura social, en dos etapas pre y postinstitucionalización hospitalaria: la muerte domesticada, la muerte propia, la muerte del otro y la muerte vedada; cuya emergencia de las ciencias médicas posibilitó su monopolización como función social bajo la lógica de lo atribuido-asignado; diría Foucault M. más allá de una posición estructural-funcionalista, que es parte de la biologización del poder o el biopoder ${ }^{5}$. Los estudios sobre la muerte progresiva en textos como "La muerte y los moribundos" de la psiquiatra suizo-norteamericana Elisabeth $\mathrm{K}-\mathrm{R}$., que derivó en toda una caracterización por etapas, de los estados psicológicos que se vivencian ante la cercanía de una muerte inminente: negación, ira, negociación, aceptación y depresión. Por otra parte en "Ideologías y Mentalidades", Michel Vovelle (1985), según Jiménez, O., R. (2009), propone un esquema de análisis que para una aproximación sociológica comprendería "una mirada vertical de la muerte que vaya "<<desde el sótano hasta el granero >>", esto es, se han de examinar los distintos niveles de la experiencia humana".

Una de las esencias contenidas en todas estas aportaciones y otras más, es que la muerte ha sido y es objeto de un pacto social de silencio. En una etapa histórica de establecimiento de la Postmodernidad, Modernidad tardía o Hipermodernidad, en donde se ha producido una importante mutación de los mecanismos de represión, antecedidos por un diseño que cualitativamente se basaba en el conservadurismo y la autoridad. Dando lugar al plusgoce como el instituido nuevo mecanismo, posibilitando la construcción de realidades paradójicas en tanto nuevo dispositivo para la producción y preservación del síntoma, siendo este no ya una denuncia 
social 6 sino una "locura personal" en mundo de comodidad y felicidad tecnológicas. En este clímax la muerte que sucede y el sufrimiento que se genera se deslegitiman, se reprivatizan, los rituales de acompañamiento del muerto y del sufriente están inscritos en el espacio de las instituciones de salud y de la burocracia.

En lo que se refiere al entorno cubano es preciso enfatizar en la repercusión transcultural que tuvo la transición hacia un nuevo modelo de relaciones sociales: el Socialismo, con el establecimiento, en una dimensión deculturación-neoculturación, del ateísmo, y su base científico y cosmovisiva en el marxismo, como legitimación ideológica y su metástasis en las preocupaciones científicos-sociales. Derivando en divorcios de temas sociales con aroma metafísico y desvelador de posturas potencialmente contradictorias, relativistas y que por tanto se cuestionaran ipso facto dicha lógica ateísta a partir de su promesa del encuentro con el bienestar humano mediante el alcance de la felicidad terrenal ${ }^{7}$. Muchas de las variables que componen el universo cognitivo de las directrices investigativas sociales $y$ humanísticas más allá de los juicios de valor con que se produzcan o recepten, alertan al sistema del desvío o no de este supraobjetivo: pobreza, desigualdad, marginalidad, emigración, familia, género, educación, organización laboral, comunidad, pareja, grupos etarios e informales, etc.

El presente estudio y los resultados obtenidos es parte de una investigación en desarrollo sobre la relación de los grupos asistenciales de salud: enfermería y médicos insertos, en unidades de atención al paciente grave; con la muerte desde la perspectiva teórica de la Representación Social. Entendiéndose que el interés radica en las condiciones inter e intrasubjetivas que mediatizadas por lo simbólico, la impronta cultural; se configuran para la inclusión y circulación del objeto muerte con arreglo a la

6. "El enfermo mental como un denunciante social", idea genialmente creada por el destacado psiquiatra argentino Enrique Pichón-Riviére. producción de comportamientos concretos potencialmente susceptibles a la estructuración de un complejo psicopatológico que va desde la Reacción al estrés agudo, Trastornos de estrés postraumático, el depresivo recurrente, de ansiedad, los no orgánicos del sueño hasta el Consumo riesgoso o el Síndrome de dependencia alcohólica. Harto preciso: teniendo en cuenta también la confluencia de otros factores.

Objetivo

Caracterizar la presencia de la muerte en las publicaciones científicas cubanas en el período 2012-2017.

\section{MÉTODO}

La metodología de investigación utilizada es cuantitativa. Se utilizaron software de visualización de información que permitió la realización de la estadística descriptiva para el análisis. Entre estos se encuentran el Excel para el tratamiento de los datos, los conteos de frecuencia y la generación de tablas y gráficos. Word Art que es una herramienta online y gratuita que permite generar nubes de etiquetas. Se emplea para graficar la concurrencia de los términos más representativos a partir de los que son más referidos en el campo de las palabras claves. Posteriormente se realizó una poda donde se eliminaron aquellos términos que no fueran repetidos al menos una vez.

Se trata de un estudio métrico, de tipo exploratorio, teniendo como precedente una amplia revisión y la entrevista a expertos en el área investigativa desde múltiples disciplinas, que arrojó de manera inicial acerca de los pocos estudios sobre la muerte como tópico de investigación. Esto nos favorece en la medida en que se "determinan tendencias, identifican áreas, ambientes, contextos y situaciones de estudio, relaciones potenciales entre variables"

7. Este es uno de los mitos heredados de la modernidad occidental, si es que en algún momento pudiera pensarse en una modernidad latinoamericana. 
(Baptista L., del P., M., Fernández C., C., Hernández S., R., 2014). Es longitudinal con carácter retrospectivo entendiéndose que se abordó el objeto de estudio a lo largo del tiempo en un período pretérito previamente delimitado. Se utilizó el análisis documental clásico, el cual consiste en la selección, recopilación, análisis y procesamiento de las fuentes documentales consultadas que sustentan el desarrollo del estudio, para la recolección de los datos necesarios de cada una de las revistas.

Además de que se dispuso de la técnica de análisis de contenido, la cual es ampliamente usada para el tratamiento de la información, inclinándose por el análisis cualitativo de contenido directo asumiéndose las unidades del discurso tal y como fueron enunciadas para identificar el predomio de subcategorías.

La batería de indicadores bibliométricos que se utilizó para dar cumplimiento al objetivo propuesto fueron: producción total, índice de concentración temática, productividad por revista, productividad anual y concurrencia de palabras claves, los que fueron operacionalizados. (Ver Tabla 1).

TABLA 1.

Batería de indicadores bibliométricos.

\begin{tabular}{|c|c|}
\hline Indicador & Operacionalización \\
\hline Producción total & $\begin{array}{l}\text { Total de registros de la } \\
\text { muestra }\end{array}$ \\
\hline $\begin{array}{l}\text { Índice de } \\
\text { concentración } \\
\text { temática }\end{array}$ & $\begin{array}{l}\text { Total de publicaciones que } \\
\text { se relacionen con la } \\
\text { temática de la muerte }\end{array}$ \\
\hline Producción anual & $\begin{array}{l}\text { Cantidad de registros por } \\
\text { cada año, según la etapa } \\
\text { comprendida en el estudio. }\end{array}$ \\
\hline $\begin{array}{l}\text { Producción por } \\
\text { revistas }\end{array}$ & $\begin{array}{l}\text { Cantidad de registros por } \\
\text { revista. }\end{array}$ \\
\hline $\begin{array}{l}\text { Co-ocurrencia de } \\
\text { palabras clave }\end{array}$ & $\begin{array}{l}\text { Relación entre las palabras } \\
\text { clave más frecuentes de la } \\
\text { muestra. }\end{array}$ \\
\hline
\end{tabular}

La muestra es de carácter intencional pautándose como criterios de selección la proyección social y humanística de la revista; que posean un alcance nacional, con respecto a la disciplina e institución científicas y al sector profesional que representan. Lo cual quiere sugiere del nivel de impacto en cuanto a la capacidad de generalización de lo publicado en términos de áreas temáticas, tópicos de interés 0 líneas de investigación. En tanto como criterios de exclusión que estuviesen inscritas a una región 0 provincia determinadas y que perteneciesen al área de las ciencias naturales y exactas, las técnicas y de economía. Hasta el momento se ha trabajado con 7 revistas y se espera ampliar la muestra: estando ceñidos al principio de saturación, por ello el rigor de presentar los resultados como preliminares; a punto de partida de obtener una visión del estado del arte en el entorno nacional.

A diferencia de otros estudios bibliométricos en donde se asume como la unidad de análisis documental el artículo original, en tanto este refleja resultados investigativos en aras de determinar avances, retrocesos o estancamientos en la producción del conocimiento científico; se asumió la publicación en bruto asegurándose de este modo ampliar el ángulo de registro. Esto implica que fue de interés también los artículos de revisión, comentarios y reflexiones, ensayos, estudios de casos; todo lo que sugiriera pistas acerca del quehacer del pensamiento científico al respecto por encontrarse abordando un dominio de la realidad poco indagado en el escenario local. Es válido precisar que en las publicaciones registradas se asumieron las que abordaron la muerte ya sea de manera directa como objeto de cognición en sí mismo - través de los distintos modos de manifestación y que propician en consecuencia formas diversas de estudio. Para ello el descriptor general definido fue muerte y como palabras claves se tuvieron en cuenta cáncer, enfermedad terminal, suicidio, final de la vida, paciente oncológico terminal, aborto, mortalidad.

\section{RESULTADOS}

A continuación una breve caracterización de la muestra. En primer lugar las que 
pertenecen a instituciones de salud, tales como La Revista Cubana de Salud y Trabajo $(\mathrm{RCST})^{8}$ que es una publicación de carácter multidisciplinario, que aborda la higiene del trabajo, los problemas de salud originados por la actividad laboral o vinculados con ella y otros temas referidos a la prevención, rehabilitación y promoción de la salud de todos los trabajadores ${ }^{9}$. Humanidades Médicas (RHM), que divulga artículos científicos de las ciencias sociales y humanísticas relacionadas con la medicina y ciencias afines ${ }^{10}$. La Revista del Hospital Psiquiátrico de La Habana (RHPH) en donde se difunde la labor científica que desarrolla Cuba en el campo de la psiquiatría, especialmente en la atención del paciente mental de larga evolución; difunde los resultados obtenidos en las actividades investigativas, asistenciales, preventivas, y educacionales en el campo de la salud mental11. Y por último la Revista Cubana de Enfermería (RCE), que presenta artículos originales 0 debatidos en simposios $y$ congresos nacionales 0 internaciones realizados en el país, referentes a la enfermería. También revisiones bibliográficas, noticias y secciones de interés general en esta especialidad ${ }^{12}$.

Las que tributan a centros del sector universitario son, Novedades en Población (NP), la cual se especializa en temas relacionados con la demografía y los estudios de población, da a conocer a su comunidad científica y a todos los interesados los últimos avances en este campo ${ }^{13}$. Alternativas Cubanas en Psicología (ACP), que se dispone para la comunicación, la promoción, la publicación de artículos, investigaciones y ensayos que

8. Se presentan esas abreviaturas en aras de facilitar la compresión de la tabla y el gráfico.

9. Catálogo de Publicaciones Seriadas Cubanas

10. Ibídem

11. Ibídem

12. Ibídem

13. Ibídem

14. Alternativas Cubanas en Psicología

15. Este concepto fue usado inicialmente en 1962 por el médico norteamericano, especialista en Oncología, Van Rensserlaer Potter cuando a solicitud de la Universidad de Dakota del Sur, fue invitado a dar una conferencia a raíz de la celebración del Centenario de la reflejan el quehacer de psicólogas y psicólogos cubanos. También publica contribuciones de profesionales de la Psicología de otros países ${ }^{14}$.

Y para finalizar la Revista Bioética ${ }^{15}$ del Centro Juan Pablo II perteneciente al sector religioso y que está dirigida a los especialistas y profesionales de especialidades afines, bioética, medicina, biología, medio ambiente, así como cualquier otra disciplina científica relacionada con la vida del hombre y su desarrollo. Transmite conocimientos actualizados con un perfil amplio. ${ }^{16}$

Entre las revistas de mayor productividad en relación a publicaciones vinculadas a la muerte destacan la revista Novedades en Población (NP) con un 11\% (9) de un total de 81 publicaciones identificadas, con un abordaje de la muerte basado en el tratamiento demográfico, en consecuencia el predominio de una comprensión estadística del fenómeno, su manifestación por variables sociodemográficas tales como: sexo, color de la piel, edad, grupo etarios; y su relación con la emigración, la esperanza de vida y la fecundidad. La muerte vista desde esta perspectiva aporta al comportamiento del binomio crecimiento - decrecimiento poblacional, en tanto alerta de sus causas actuantes, así como del establecimiento de un patrón en una u otra dirección.

Seguidamente la Revista de Bioética del Centro Juan Pablo II (RBCJPII) que presenta un $9 \%(7)$ de 79 publicaciones registradas. Reconociéndose como tendencia publicaciones sobre el estatus ontológico de la vida y la muerte, de ahí que sus estudios

\footnotetext{
Ley Morril. Pero no es hasta la década de los $70^{\prime}$ donde aparece como una propuesta científica con mayor solidez explicativa por medios de sus dos obras clásicas: "Bioética. La ciencia de la supervivencia" y posteriormente "Bioética. Puente hacia el futuro". Según González, L., Ingrid (2012) "en la acepción potteriana, la nueva disciplina se concibe como un compromiso fundamental de integración del saber científico y los valores humanos; es en este sentido un saber nuevo, de unificación que precisa de ser en la medida que supere a la antigua concepción dicotómica entre conocimiento y valor, la ciencia y las humanidades, establecidas por la tradición occidental." 16. Óp. Cit.
} 
estén enfocados en el aborto como problemática acerca de la toma de decisiones sobre la vida y que cosmovisión sustenta su inicio, si desde la vida intrauterina o desde el momento en que se inserta en el mundo propiamente social y le es atribuible la categoría de persona; el acompañamiento durante los cuidados paliativo y su pertinencia o la interrupción de procedimientos tecnológicos que extienden inútilmente determinas funciones vitales aun cuando el proceso de muerte es irreversible, haciéndose énfasis en la concepción de muerte digna. Los estudios desde estos análisis dan cuenta de la relación con la muerte y los aportes de la Bioética para su comprensión, incluso desde una perspectiva epistemológica, visualizando cuestionamientos sobre la racionalidad impuestas por la modernidad en marco del mito de progreso y desarrollo humano teniendo como vehículo a las ciencias.

La Revista del Hospital Psiquiátrico de la Habana (RHPH) produjo, de 181 publicaciones registradas, un $7 \%$ (12) relacionadas con la muerte predominando los estudios suicidiológicos con énfasis en los factores de riesgo a nivel familiar $y$ personal, la población vulnerable, la comisión del intento. Llama la atención que la mayoría de los estudios provengan de la zona oriental del país, específicamente de la provincia de Granma siendo la adolescencia el grupo de investigación de interés. En menor medida se tienen los estudio psicooncológicos manejándose variables como claudicación familiar y paciente oncológico. De modo general esto obedece al perfil clínico de la psiquiatría, aunque el estudio pionero sobre este manifestación de la muerte proviene de la Sociología, específicamente de la obra del sociólogo francés Durkheim E. titulada "El Suicidio".

La Revista Cubana de Salud y Trabajo produjo un $4 \%$ (8) de publicaciones relacionadas con la muerte de 188 registradas. Caracterizándose por los estudios de mortalidad y su vinculación con la seguridad laboral y la productividad; la muerte como interruptora de la vida laboral, su incidencia en la eficiencia en el trabajo, en el aprovechamiento de las capacidades productivas en términos de aporte personal y social.

En la Revista Humanidades Médicas (RHM) se registraron 245 publicaciones, de ellas un 3\% (7) están vinculadas a la muerte, pudiéndose establecer como patrón la presencia de publicaciones cuyas temáticas son los cuidados paliativos y la perspectiva bioética relacionada con la muerte. De modo disperso se publica sobre la problemática del aborto selectivo, la morbi-mortalidad en los procesos quirúrgicos.

La Revista Cubana de Enfermería (RCE) con 288 publicaciones presenta un $2 \%$ (5) de productividad de contenidos que se vinculen a la muerte, entre los que no se pudo identificar un patrón estable de publicaciones al respecto. Por lo que no se apreció un tópico predominante, identificándose publicaciones sobre la mortalidad infantil, prevención del cáncer, la humanización de la asistencia al paciente crítico, sobre el cuidado. Esta constituye la revista de mayor productividad en términos de cantidad de publicaciones y en la que se aprecia una relación inversamente proporcional con respecto a la cantidad de publicaciones vinculadas a la muerte. Suscita la atención siendo la enfermería una de las profesiones de mayor vinculación con el paciente, y su importancia en contextos específicos del ámbito hospitalario donde la muerte prevalece.

Destacar en este apartado analítico a la revista Alternativas Cubanas en Psicología, donde de las 165 publicaciones registradas solamente 1 publicación se relaciona con la muerte, y es desde la perspectiva de la prevención del suicidio en el marco de los aportes de la Psicología Educativa al respecto, proveniente por demás del oriente del país. No es ocioso traer a colación el interesante artículo publicado por un 
sociólogo inglés llamado Walter en la década de los 90", titulado "Los sociólogos nunca mueren", para sugerir acerca de la ausencia de la muerte ${ }^{17}$ como variable de investigación en un medio de divulgación científica que según se refiere se erige para reflejar el quehacer de los profesionales de la Psicología en el país, aun cuando se realizase un análisis más exhaustivo sobre la disciplinas con mayor presencia. Correspondería entonces algunos cuestionamientos acerca de las variables investigativas que dan vida a disciplinas como Psicología Clínica y Psicología de la Salud en el ámbito local por un lado y por el otro el impacto de las mismas en las líneas de divulgación de la revista.

Agrupando las revistas por sectores profesionales, nótese que la Revista del Hospital Psiquiátrico de La Habana (RHPH), fue la que más produjo con un $7 \%$ de publicaciones relacionadas con la muerte dentro de las que pertenecen al sector de la salud, encontrándose como las menos productivas a la Revista Cubana Enfermería (RCE) con un $2 \%$ y la Revista Humanidades Médicas (RHM) con un 3\% respectivamente. Atendiéndose a la productividad según las revistas con más de 150 publicaciones registradas, la Revista del Hospital Psiquiátrico de La Habana (RHPH) se mantiene como la de mayor productividad, siendo entonces la más improductiva la revista Alternativas Cubanas en Psicología (ACP) con solamente una publicación. Sin embargo las revistas con menor cantidad de publicaciones, tales como la Revista de Bioética del Centro Juan Pablo II (RBCJPII) y Novedades en Población (NP) mostraron una apreciable productividad en relación con el resto.

Desde una visión global, de las 1227 publicaciones registradas se identificó un índice de concentración temática de un 4\% (49), equivalente a una notable baja productividad de estas revistas en cuanto a publicaciones que guarden relación con la muerte. (Ver Tabla 2).

TABLA 2.

Productividad por revistas en cuanto a cantidad de publicaciones y las relacionadas con la muerte.

\begin{tabular}{lccc}
\hline Revistas & $\begin{array}{c}\text { Relacionadas } \\
\text { con la muerte }\end{array}$ & $\begin{array}{c}\text { Cantidad } \\
\text { en } \%\end{array}$ & Publicaciones \\
\hline RHPH & 12 & 7 & 181 \\
RCST & 8 & 4 & 188 \\
RCE & 5 & 2 & 288 \\
RHM & 7 & 3 & 245 \\
ACP & 1 & 0 & 165 \\
RCBJPI & 7 & 9 & 79 \\
RNP & 9 & 11 & 81 \\
Total & 48 & 4 & 1227 \\
\hline
\end{tabular}

En correspondencia con la distribución de la productividad por años y revistas, las que se mantuvieron con una producción estable, entendiendo por esto que sus publicaciones en relacionadas con la muerte tuvieron una cierta periodicidad, se encuentran: la revista Novedades en Población que produjo de manera constante a lo largo del período comprendido, en donde destacó el 2014 (3) como el de más productividad identificándose los estudios sobre la mortalidad infantil, la Revista del Hospital Psiquiátrico de La Habana (RHPH) que sostuvo una continuidad desde el año 2012 hasta el año de 2016, siendo el 2015 (4) su año más productivo en donde las publicaciones que predominaron fueron nuevamente los estudios suicidiológicos; seguidamente la Revista Cubana de Salud y Trabajo (RCST) que excepto el año 2102 mantuvo una producción anual llegando hasta el 2017, identificándose al 2014 (3) como el año más productivo manifestándose en este particular los estudios que comprenden la relación de la muerte con la vida laboralmente productiva. En el caso de las revistas que no mantuvieron una producción estable también se efectuó la identificación de sus años más productivos, destacando para la Revista de Bioética del

17. Aunque según uno de los cinco axiomas de la Teoría de la Comunicación Humana de Paul Watzlawick, es imposible no comunicar. 
Centro Juan Pablo II (RBCJPII), el año 2012 (4) como el de más publicaciones, referidas estas a la problemática de la muerte digna, la gestión del occiso y el aborto. Para la Revista Cubana de Enfermería (RCE) el año más productivo fue el 2013 (3), con publicaciones sobre mortalidad neonatal, cuidados paliativos y la humanización de la atención al paciente grave. Sobre la Revista
Humanidades Médicas, el 2012 (3) fue el de más alta productividad a partir de las publicaciones sobre la muerte encefálica desde un enfoque social, los cuidados paliativos y los valores ante el enfermo terminal. No se toma en cuenta la revista Alternativas Cubanas en Psicología por registrarse una sola publicación en el período delimitado (Ver Gráfico 1).

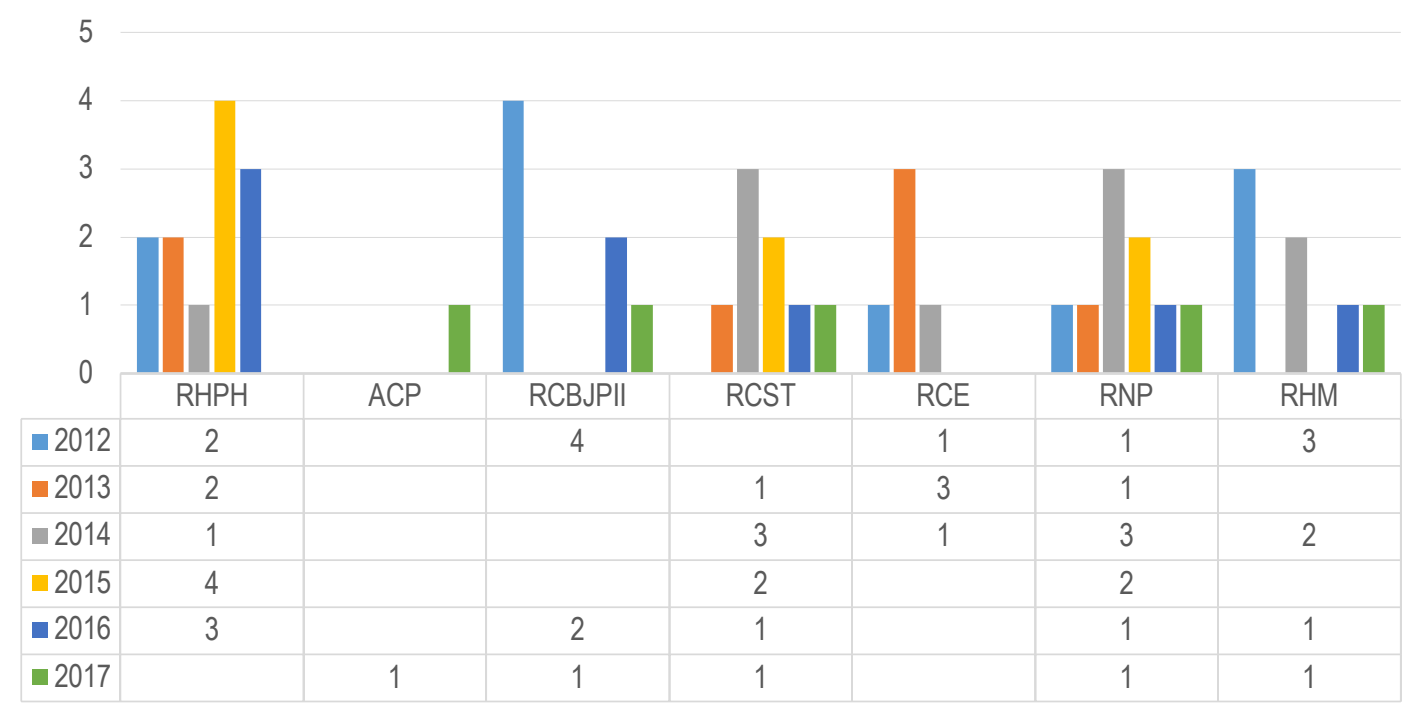

GRÁFICO 1.

Distribución de la productividad por año y revista (período 2012 - 2017).

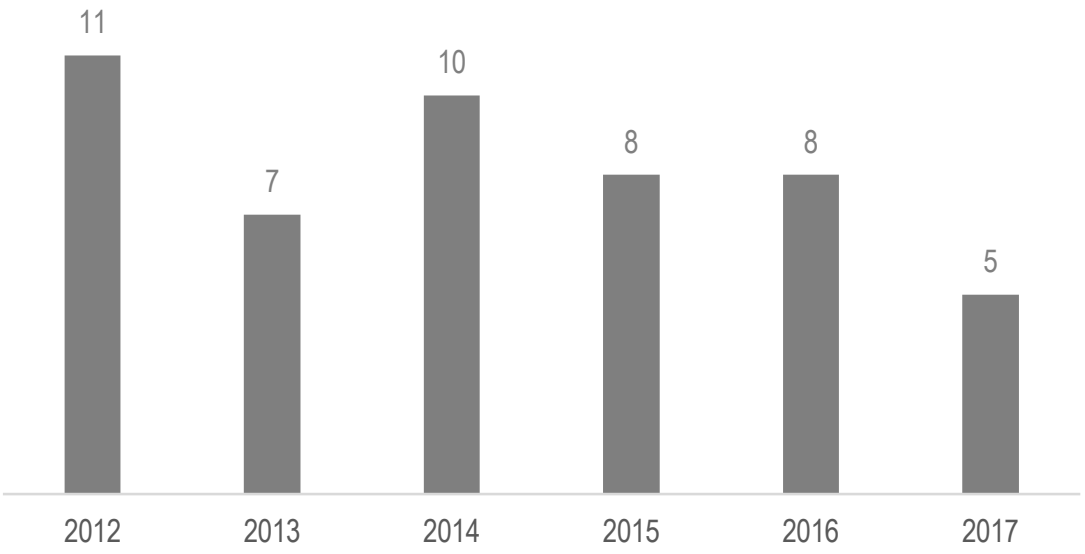

GRÁFICO 2.

Productividad anual (período 2012 - 2017).

Atendiendo a la productividad anual, el año 2012 fue el más productivo por el aporte de revistas como la Revista de Bioética del Centro Juan Pablo II con 4 publicaciones, seguida de la Revista Humanidades Médicas con 3 publicaciones. Posteriormente el año 2014, por la incidencia de la Revista de Salud y Trabajo con 3 publicaciones al igual que la revista Novedades en Población. Y en ese orden están los años 2015 y 2016 con similar 
producción anual: 8 publicaciones Destaca nuevamente la Revista del Hospital Psiquiátrico de La Habana en cuanto a aportes con 4 y 3 publicaciones respectivamente. Finalmente cierra el año 2017 como el de menor productividad, señalándose a las revistas de Enfermería y del Hospital Psiquiátrico de La Habana como las únicas que no produjeron publicaciones relacionadas con la muerte. También es preciso acotar que con respecto a la Revista del Hospital Psiquiátrico de La Habana, no se pudo registrar la información de los volúmenes 2 y 3 de ese año por no encontrarse publicados, por lo que el argumento anteriormente expuesto es relativo hasta que se realice la correspondiente revisión. (Ver Gráficos 2 y 1 en ese orden).

En cuanto a la concurrencia de los términos relacionados con la muerte analizándose su presencia y frecuencia en el campo de las palabras claves entre las que más predominan se encuentra suicidio y mortalidad, lo que da cuenta de las perspectivas de estudio imperantes sobre la muerte. La primera enmarcada en lo que podría denominarse investigaciones suicidiológicas, con un tratamiento fundamentalmente clínico en el área de las ciencias de la salud mental, con el precedente sociocultural del cual es depositario este tipo de manifestación de la muerte. El término mortalidad posee una connotación demográfica de ahí su tratamiento estadístico, esta perspectiva posee una larga tradición investigativa, acá la muerte es un factor de equilibrio 0 desequilibrio, en dependencia de la participación de otras condiciones, de los patrones de reproducción, distribuidos atendiendo a las variables demográficas, incluso aquellas que en determinadas circunstancias históricas se torne estratégica para la continuidad del sistema social (Ver figura 1).

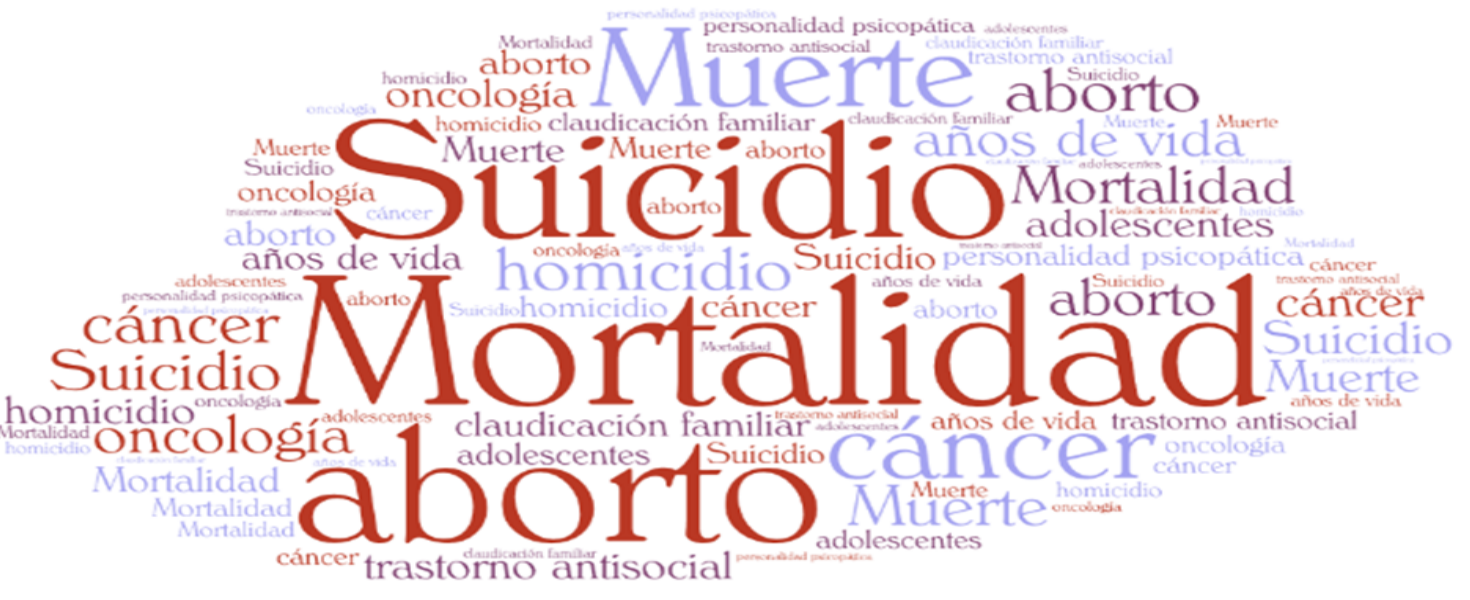

FIGURA 1.

Distribución de la productividad por año y revista.

\section{CONCLUSIONES}

El grupo de revistas analizadas se caracteriza por una baja productividad de publicaciones relacionadas con la muerte, predominando aquellos tópicos que se corresponde con el ajuste de sus emisores a la división social del sistema de ciencias del país. Lo que constituye una alerta sobre el hecho fáctico de que la muerte no es susceptibles de ser un objeto de investigación con capacidad potencial para la apertura de nuevas zonas de sentido que ofrezcan visiones distintas sobre lo humano. 
Su pertinencia ontológica sugiere interrogantes sobre la comunidad de profesionales de las ciencias en cuanto al modelo de relacionamiento que reproducen en su comprensión científica de la realidad con arreglo al imprinting cultural ${ }^{18}$ que los gobierna. Resaltando el argumento, por más obvio que parezca, que la incidencia de la muerte en una sociedad atraviesa necesariamente las zonas de la inter e intrasubjetividad.

\section{REFERENCIAS}

Baptista, L. P.; Fernández, C. C. y Hernández, S. R. (2006). Metodología de la investigación (4a ed.). McGraw-Hill Interamericana. México.

Carlos, G. J. (2017) "La vida, la muerte y la medicina en la Unidad de Terapia Intensiva. Versión electrónica. Recuperado de www.infomedicos.org/etica-medica/lavida-la-muerte-y-la-medicina-en-launidad-de-terapia-intensiva/

Colectivo de autores (2008). Ansiedad y actitudes ante la muerte: revisión y caracterización en un grupo heterogéneo de profesionales que se capacita en cuidados paliativos. Revista Pensamiento Psicológico, 4,10.

Colectivo de autores (2014). Catálogo de Publicaciones Seriadas Cubanas. Dirección de Publicaciones Periódicas del Instituto Cubano del Libro. Ministerio de Cultura: La Habana. Cuba.

Colectivo de autores (2001). Tercer Glosario Cubano de Psiquiatría. Hospital Psiquiátrico de La Habana. Cuba

Delgado, D. J. C. (2007). Hacia un nuevo saber. La bioética en la revolución contemporánea del saber. Publicaciones Acuario, Centro Félix Varela. La Habana. Cuba

Fajardo Egües, Y. (2016). Estudio Bibliométrico de la Revista Cubana de Higiene y Epidemiología en el período

18. Según Morín E. (2003) "determina la falta de atención selectiva, que nos hace despreciar todo lo que no vaya en el sentido de nuestras creencias, el rechazo eliminatorio, que nos hace rehusar
1995-2014. Trabajo de Diploma. Departamento de Ciencias de la Información. Facultad de Matemática, Física y Computación, Universidad Central "Marta Abreu". Villa Clara: Cuba González, L., Ingrid (2012). Bioética Global y ética de la complejidad. Tesis de maestría. Centro de Estudio de Salud y Bienestar Humano, Universidad de La Habana. Cuba

González Rey, F. (1997).Epistemología cualitativa y subjetividad. Editorial Pueblo y Educación. La Habana. Cuba Jiménez Aboitiz, R. (2009). ¿De la muerte (de) negada a la muerte reivindicada? Análisis de la muerte en la sociedad española actual: muerte sufrida, muerte vivida y discursos sobre la muerte. Tesis de doctorado. Departamento de Sociología y Trabajo Social, Universidad de Valladolid. España.

Lovesio, C. (2011). La lucha de los médicos que conviven con la muerte en la terapia intensiva". Versión electrónica. Recuperado de: www.infobae.com/2011/08/24/601561la-lucha-los-medicos-que-conviven-lamuerte- las-terapias-intensivas

Morín, E. (1986). El método. El conocimiento del conocimiento. Vol. III. Cátedra. Madrid. España (2003). El hombre y la muerte (4ta ed.). Editorial Kairós. Barcelona: España

Perera, M. (2005). Sistematización crítica de la teoría de las Representaciones Sociales. Tesis de doctorado. Centro de Investigaciones Psicológicas y Sociológicas. La Habana. Cuba.

Philippe, A. (2000). Historia de la muerte en Occidente. Desde la Edad Media hasta nuestros días. Acantilado Quaderns Crema, S.A.U. Barcelona. España. toda información inadecuada a nuestras convicciones o toda objeción procedente de una fuente reputada de mala." 